\title{
Properties of nanocrystalline Si layers embedded in structure of solar cell
}

\author{
Stanislav Jurečka ${ }^{*}$, Kentaro Imamura**, \\ Taketoshi Matsumoto $^{* *}$, Hikaru Kobayashi ${ }^{* *}$
}

\begin{abstract}
Suppression of spectral reflectance from the surface of solar cell is necessary for achieving a high energy conversion efficiency. We developed a simple method for forming nanocrystalline layers with ultralow reflectance in a broad range of wavelengths. The method is based on metal assisted etching of the silicon surface. In this work, we prepared Si solar cell structures with embedded nanocrystalline layers. The microstructure of embedded layer depends on the etching conditions. We examined the microstructure of the etched layers by a transmission electron microscope and analysed the experimental images by statistical and Fourier methods. The obtained results provide information on the applied treatment operations and can be used to optimize the solar cell forming procedure.
\end{abstract}

K e y w or ds: semiconductor, silicon solar cell, microstructure, Fourier analysis

\section{Introduction}

Suppression of light reflectance from the sample surface is necessary for achieving a high energy conversion efficiency of Si solar cells. Formation of antireflection layers and texturization of Si surface are commonly used for these purposes. The development of new technologies is dominantly motivated by achieving a lower cost of electricity production. Forming of antireflection layers increases the cost of the prepared structures. The spectral reflectance is in this case suitably decreased only in limited ranges of wavelengths and for limited incidence angles. Pyramidal textures formed on Si surface can be used to improve the light trapping [1-6]. The spectral reflectance of a pyramidal texture on Si decreases to $10 \%$. Another way of production of $\mathrm{Si}$ structures with a low spectral reflectance is fabrication of porous structures [79]. Porous structures are usually nonuniform and due to the thickness of the porous layer it is difficult to form a high-quality pn-junction for the solar cell.

A simple method of forming nanocrystalline layers with ultralow reflectance in a broad range of wavelengths has been developed in Osaka University [10-13]. The method is based on metal assisted etching of Si. A Pt mesh immersed in $\mathrm{HF}$ and $\mathrm{H}_{2} \mathrm{O}_{2}$ solution is in contact with the Si surface and the mesh structure is transferred on the etched surface (surface structure chemical transfer method, SSCT). Due to the nonuniform dissolution, the SSCT treatment forms porous nanocrystalline layers with a gradient of pore density [6]. The ultralow spectral reflectance of nanostructured Si layer/crystalline $\mathrm{Si}$ structure results therefore from the depth gradient of the refractive index increasing with the thickness of the SSCT layer. In this work, we prepared solar cell structures with embedded SSCT layers. The microstructure of the solar cells with SSCT layers formed by various technological operations was experimentally measured by the transmission electron microscope (TEM). The cross-sectional TEM image contains important information about the microstructure development during individual steps of forming operations. We analysed the properties of embedded SSCT layers by studying statistical properties of the two-dimensional Fourier transform of a selected area in the TEM image with the SSCT structure. Results of this analysis provide reliable information necessary for optimization of the SSCT forming procedure.

\section{Theoretical part}

The Fourier transform is an important image processing tool used to decompose an image into its harmonic components. Two-dimensional (2D) image function $f(m, n)$ defined on an $M \times N$ grid selected at the TEM image is in our approach transformed by the $2 \mathrm{D}$ discrete Fourier transform [14]

$$
\begin{array}{r}
F(k, l)=\frac{1}{\sqrt{M N}} \sum_{m=0}^{M-1} \sum_{n=0}^{N-1} f(m, n) e^{-2 \pi i\left(\frac{m k}{M}-\frac{n l}{N}\right)} \\
k=0,1, \ldots M-1, \quad l=0,1, \ldots N-1
\end{array}
$$

The structure of the Fourier domain $F(k, l)$ is reproduced graphically in a 3D plot (coded into grey scale or in color) and studied by statistical methods. We analysed

\footnotetext{
* Institute of Aurel Stodola, University of Žilina, Nálepku 1390, 03101 Liptovský Mikuláš, Slovakia, jurecka@lm.uniza.sk, ** Institute of Scientific and Industrial Research, Osaka University and CREST, Japan Science and Technology Agency, Mihogaoka 8-1, Ibaraki, Osaka 567-0047, Japan
} 

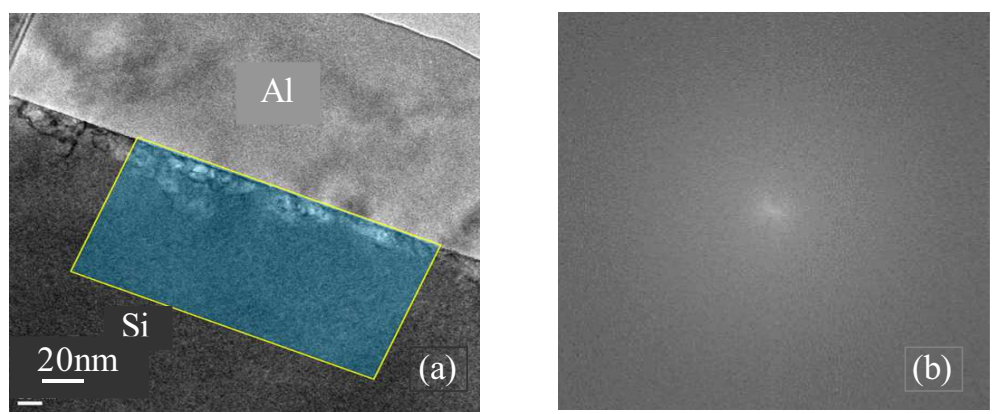

Fig. 1. (a) - TEM image of the Si/SSCT structure with selected area for the microstructure analysis, (b) - 2D DFT map of selected area in the TEM image of the SSCT layer embedded in a solar cell

Table 1. Solar cell structures with embedded SSCT layer

\begin{tabular}{lc}
\hline Sample & $\begin{array}{c}\text { Etching time } \\
(\mathrm{s})\end{array}$ \\
\hline ET05 & 5 \\
ET10 & 10 \\
ET15 & 15 \\
ET20 & 20 \\
ET25 & 25 \\
ET30 & 30 \\
\hline
\end{tabular}

the distribution of the Fourier domain values along selected directions in the $F(k, l)$ space and evaluated the coefficients of skewness and kurtosis. These statistical moments are defined by central moments of the analysed values [15]. The $k$-th central moment of the random variable $X$ is defined by

$$
\mu_{k}=E(X-\mu)^{k}, \quad k=1,2, \ldots
$$

where $\mu=E(X)$ is the mean of $X$. The second central moment $\mu_{2}$ is called the variance and is a measure of the variability of $X$ values. The coefficient of skewness is

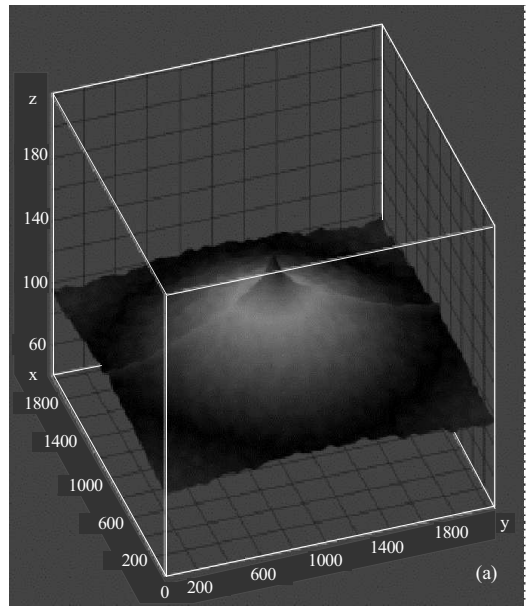

defined by equation

$$
S_{k u}=\frac{\mu_{3}}{\mu_{2}^{3 / 2}}
$$

This is a measure of skewness of distribution $X$. For positive value of $S_{k u}$ the distribution $X$ has long right tail (distribution is skewed to right). Value

$$
S_{k u}=\frac{\mu_{4}}{\mu_{2}}
$$

is called the coefficient of kurtosis. It is a scale and location invariant measure of the degree of peakedness of the probability density curve. Values of $S_{k u}$ and $F(k, l)$ significantly change in correspondence with the applied solar cell forming operations and indicate the degree of modification of the SSCT microstructure.

\section{Experimental part}

The SSCT layers in a structure of solar cells have been formed on Si substrate for a set of etching times described in Table 1. Transmission electron micrograph measurements of the cross-section microstructure of the solar cell with embedded SSCT layer were carried out using a JEOL EM-3000F microscope with the incident electron energy of $300 \mathrm{keV}$.

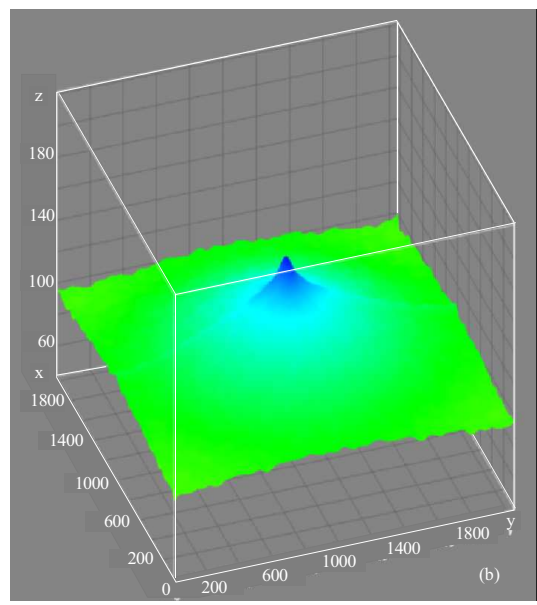

Fig. 2. 2D DFT map of selected area in the TEM image with the SSCT layer : (a) - $2 \mathrm{D}$ DFT values represented in the grey scale, and (b) - in the color scale 

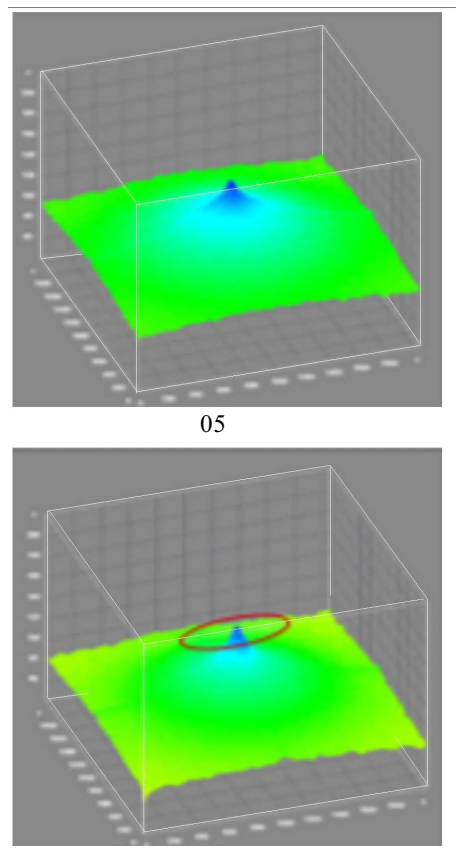

20

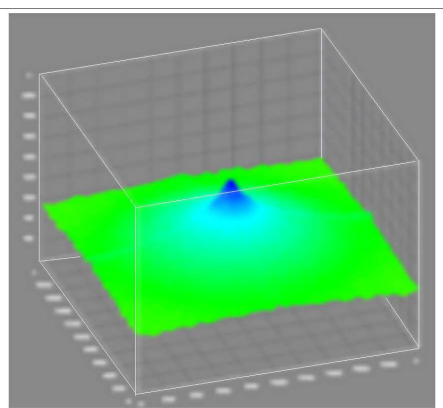

10

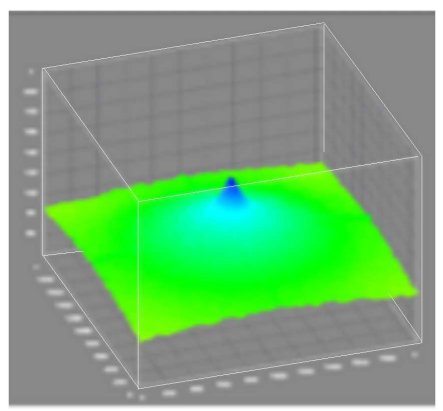

25
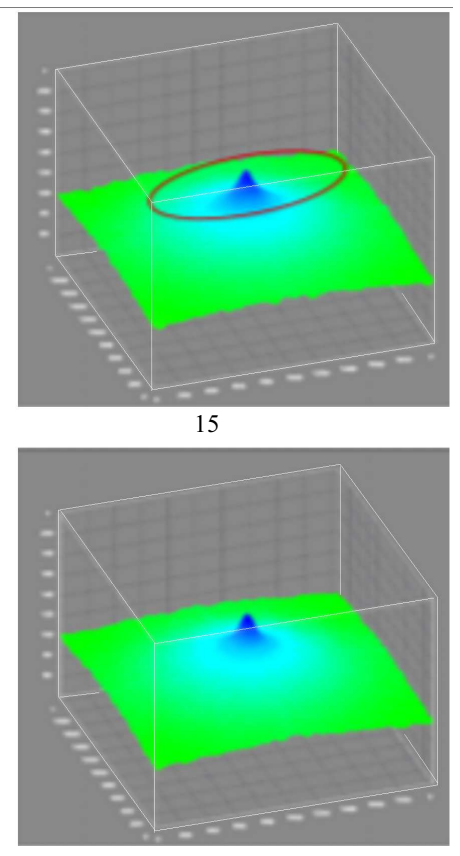

30

Fig. 3. 2D DFT map of area in the TEM image with the SSCT layer embedded in solar cell, with SSCT layer formed for 5, 10, 15, 20, 25,30 seconds

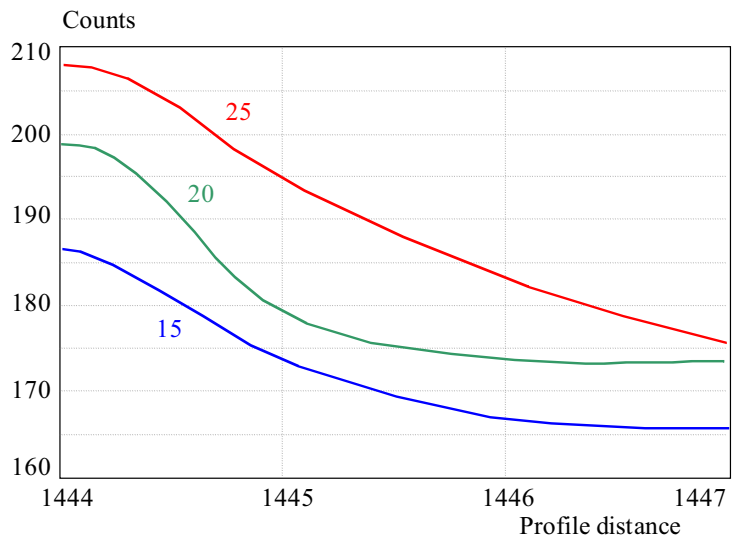

Fig. 4. Detail of the central part of profile shape along diagonal cross - section in the $1^{\text {st }}$ quadrant of the $2 \mathrm{D}$ DFT domain, SSCT layer has been formed for 15,20 and 25 seconds

\section{Results and discussion}

TEM images of SSCT layers embedded in the structure of the solar cell contain information about the layer thickness and about the microstructural properties influenced by individual steps of technological operations. In the analysed TEM images we selected a rectangular area containing information about the microstructure of the SSCT layer. We used identical dimension of selected areas for the whole set of samples as can be seen in Fig. 1a. The values in the selected area were transformed by the 2D DFT using Eq. 1 and represented in a grayscale 2D image (Fig. 1b), grayscale 3D image (Fig. 2a) and color $3 \mathrm{D}$ image (Fig. 2b).

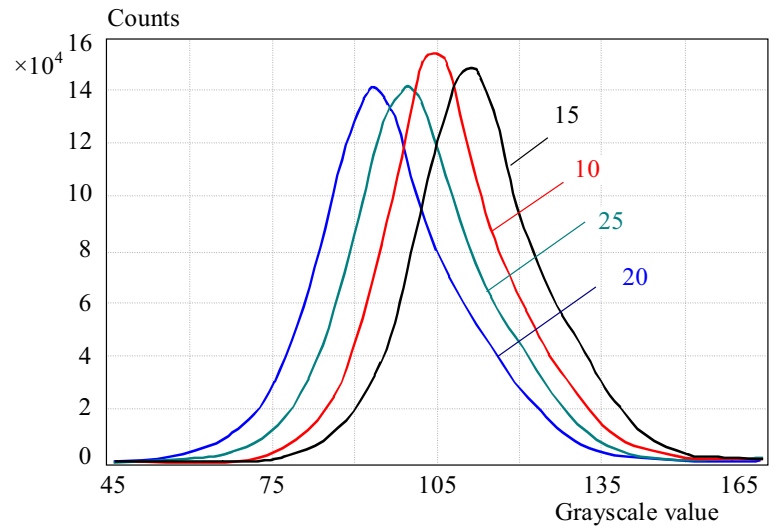

Fig. 5. Histogram of values in the Fourier domain along diagonal cross - section in the 1st quadrant of the 2D DFT domain, with SSCT layer formed for $10,15,20$ and 25 seconds

Space frequencies shown in the 2D DFT domain reflect the development of the SSCT microstructure with the prolongation of the etching time. In Fig. 1(b) we observe a bright central zone of the Fourier domain. The properties of this area are very sensitive to changes in the analysed microstructure.

This is nicely documented by Fig. 3 and 4 . We observed increasing values and changes of the central peak shape. Significant changes in the central zone can be seen in the ranges between 15, 20 and 25 second of SSCT etching (in Fig. 3 marked by circles). In Fig. 4, the profile values along the diagonal of the 1st quadrant of $2 \mathrm{D}$ DFT domain are shown. We observed a significant change of the profile function for etching time 20 seconds again. The distribution of the values for the structure etched for 


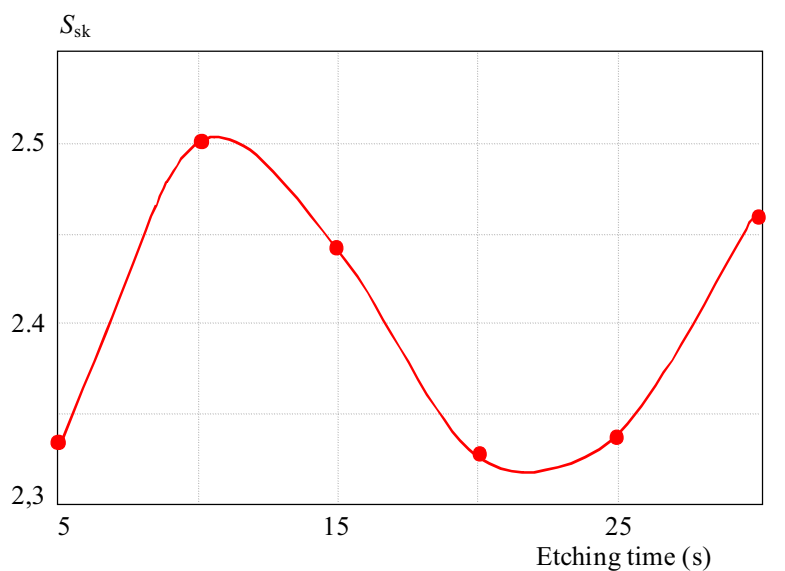

Fig. 6. The coefficient of skewness $S_{\text {sk }}$ for distributions represented by histograms in Fig. 5

20 seconds is narrower. The properties of particle size distribution in the SSCT layer are different for this etching time.

In Fig. 5, the histograms of values distributed along the diagonal of the $1^{\text {st }}$ quadrant of the $2 \mathrm{D}$ DFT domain are shown.. Changes of the histogram shape are determined by changes of particle size distribution in the SSCT layer during etching. In the first steps of the SSCT etching values in the 2D DFT domain represented by histogram shift towards the higher values (up to 15 seconds of etching). This trend drastically changes between 15-20 seconds and relaxes with a further prolongation of etching.

The properties of distributions represented by these histograms are influenced by the development of the SSCT microstructure during etching. Coefficients of skewness $S_{s k}$ and kurtosis $S_{k u}$ computed for these distributions by using (2) and (3) are shown in Fig. 6 and Fig. 7.

The coefficient of skewness increases in the initial stages of etching. With prolongation of the etching time it scales down and reaches minimal values for etching times around 20 seconds. The $S_{S k}$ values increase after this time again. This behaviour agrees with the development of particle size distribution in the SSCT layers during etching represented by histograms in Fig. 5. $S_{s k}$ values indicate asymmetrical distribution of values in the Fourier domain in comparison with the normal distribution. These values are asymmetric, skewed right and indicate a significant presence of outliers in the right tail of the distribution. This asymmetry increases during etching and reaches less critical values for etching time of 20 seconds. The particle size distribution fabricated during the SSCT etching has lower asymmetry for etching time of 20 seconds in comparison with other SSCT fabricated structures.

Also the coefficient of kurtosis increases in the initial stages of etching. Its behaviour is very similar to the $S_{s k}$ values in Fig. 6. $S_{k u}$ coefficient is a measure of peakedness of analysed distribution. Resulting values of $S_{k u}$ indicate that the distribution in the Fourier domain may be considered as not normal (for normal distribution $S_{k u}=0$ ). Peakedness decreases for etching time of 20 seconds.

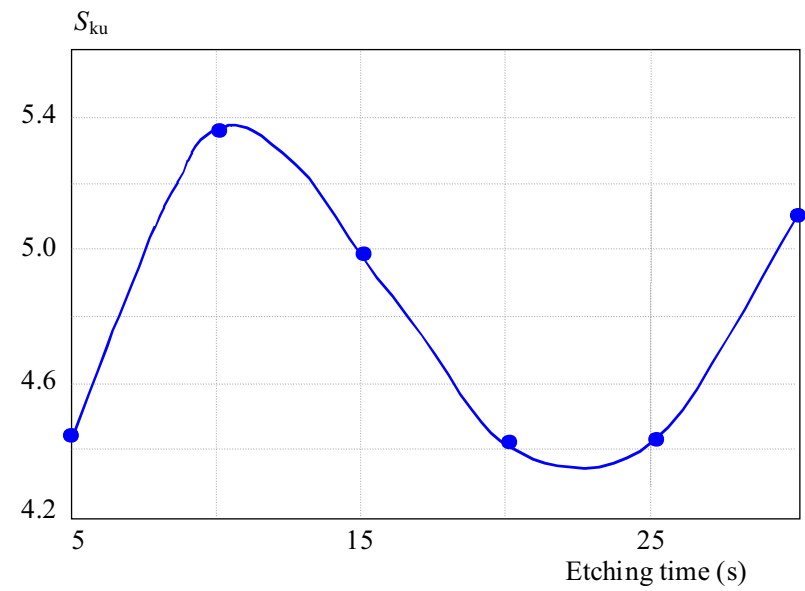

Fig. 7. The coefficient of kurtosis $S_{\mathrm{ku}}$ for distributions represented by histograms in Fig. 5

Changes in the SSCT microstructure during the first 20 seconds of etching are substantial for obtaining the required lowering of the spectral reflectance, which is crucial for the solar cell performance. Particle size distribution develops very intensively in the initial stages of etching. During the SSCT etching time of 20 seconds, the formed microstructure features reduce the spectral reflectance in a broad range of wavelengths.

\section{Conclusion}

Special nanostructured SSCT layers with an ultralow spectral reflectance were formed on a Si surface. The fabricated layers were embedded in the structure of a Si solar cell. The microstructure of nanocrystalline layers formed by various technological operations was experimentally examined by TEM. We analysed the properties of formed SSCT microstructures by 2D DFT transform of selected regions in a cross-sectional TEM image. Distributions of the values in the Fourier domain were analysed by statistical methods. Results of this theoretical approach are consistent, provide reliable information about the intensity of structure modification during the etching procedure and can be used for optimization of the SSCT layer forming steps.

\section{Acknowledgements}

The results were developed within the research projects supported by the Japan Society for the Promotion of Science and Osaka University research project Analysis of Properties of the SSCT Layers, Kobayashi Laboratories, ISIR, Osaka University, Japan. The work was supported by grant of Science and Technology Assistance Agency APVV-15-0152, Scientific Grant Agency VEGA 1/0676/17 and 1/0076/15, Centre of Excellence of Power Electronics Systems and Materials ITMS 26220120003 and ITMS 26220120046, and project ITMS 26210120021. 


\section{REFERENCES}

[1] P. Papet, O. Nichiporuk, A. Kaminski, Y. Rozier, J. Kraiem, J.-F. Lelievre, A. Fave, A. Chaumartin and M. Lemiti, "Pyramidal texturing of silicon solar cell with TMAH chemical anisotropic etching", Solar Energy, Mater.Sol.Cells, vol. 90, 2006, pp. 2319

[2] B. Stegemann, J. Kegel, L. Korte and H. Angermann, "Surface Optimization of Random Pyramid Textured Silicon Substrates for Improving Heterojunction Solar Cells", Solid State Phenomena, vol. 255, 2016, pp. 338-343.

[3] A. Angermann, J. Rappich, L. Korte, I. Sieber, E. Conrad, M. Schmidt, K. Hübener, J. Polte and J. Hauschild, "Wet-chemical passivation of atomically flat and structured silicon substrates for solar cell application", Applied Surface Science, vol. 254, 2008, pp. 3615-3625.

[4] H. Kim, S. Park, S. M. Kim, S. Kim, Y. D. Kim, S. J. Tark and D. Kim, "Influence of surface texturing conditions on crystalline silicon solar cell performance", Curr. Appl. Phys. vol. 13, 2013, S34.

[5] S. Jurečka, H. Angermann, H. Kobayashi, M. Takahashi and E. Pinčík, "Multifractal analysis of textured silicon surfaces", Applied Surface Science, vol. 301, 2014, pp. 46-50.

[6] S. Jurečka, T. Matsumoto, K. Imamura and H. Kobayashi, "Multifractal analysis and optical properties of nanostructured silicon layers", Applied Surface Science, vol. 395, 2017, pp. 150-156.

[7] J. Oh, H. Yuan and H. Branz, "An 18. 2\%-efficient black-silicon solar cell achieved through control of carrier recombination nanostructures", Nat. Nanotechnol. vol. 7, 2012, pp. 743.

[8] E. S. Kooij and D. Vanmaekelbergh, "Catalysis and pore initiation the anodicdissolution of silicon HF", J. Electrochem. Soc. vol. 144 , no. 4, 1997, pp. 1296-1301.

[9] K. Tsujino, M. Matsumura and Y. Nishimoto, "Texturization of multicrystalline silicon wafers for solar cells by chemical treatment using metallic catalyst", Sol. Energy Mater. Sol. Cells, vol. 90, 2006, pp. 100.

[10] K. Imamura, Jr. F. C. Franco, T. Matsumoto and H. Kobayashi, "Ultralow reflectivity polycrystalline silicon surfaces formed by surface structure chemical transfer method", Appl. Phys. Lett. vol. 103, 2013, 013110.

[11] K. Imamura, T. Nonaka, Y. Onitsuka, D. Irishika and H. Kobayashi, "Light trapping of crystalline Si solar cells by use of nanocrystalline Si layer plus pyramidal texture", Applied Surface Science, 2016, http://dx.doi.org/10.1016/j.apsusc.2016.04. 132.

[12] D. Irishika, K. Imamura and H. Kobayashi, "Sol. Energy Mat. Sol. Cells", vol. 141, 2015, pp. 1-6.

[13] K. Imamura, T. Nonaka, D. Irishika and H. Kobayashi, ECSD Solid State Lett. vol. 4, 2015, Q63-Q65.

[14] D. Sundararajan, The Discrete Fourier Transform, Theory, Algorithms and Applications, World Scientific Publishing, Singapore, 2001.

Received 23 April 2017

Stanislav Jurečka (Assoc Prof, Dr, PhD) received the Magister degree in mathematics and physics and the Dr degree from the Comenius University, Bratislava, Slovakia in 1981. From 1984 to 2002 he worked in Department of Physics, Academy of Armed Forces in Liptovský Mikuláš, Slovakia. Since 2002 he has been a Lecturer in the Institute of Aurel Stodola, University of Žilina, Liptovský Mikuláš, Slovakia. He received the $\mathrm{PhD}$ degree from Technical University in Košice,
Slovakia in 2005. He has been engaged in research of the device microstructure properties, charge transport processes and optical properties of semiconductor-dielectric systems. His research activities are targeted on material analysis, silicon solar cells, methods of improving the conversion efficiency, analysis of the structure passivation, density of defect states, analysis of silicon surface nanostructuring in connection with the optical properties.

Kentaro Imamura (Assist Prof, Sci, PhD) received his $\mathrm{MSc}$ and $\mathrm{PhD}$ degrees in chemistry from Osaka University, Osaka, Japan, in 2008 and 2012, respectively. From 2008 to 2012, he was a Researcher in Corporate Research \& Development group of Sharp Corporation, Nara, Japan where he engaged in development of LCD devices. Since 2012, he has been an Assistant professor in the Institute of Science and Industrial Research, Osaka University, Osaka, Japan. His research interests are Si solar cells, especially new chemical methods to improve conversion efficiencies such as surface structure chemical transfer (SSCT) method for achievement of ultralow reflectance and nitric acid oxidation of silicon (NAOS) method for surface passivation, and fabrication and application of nano-Si materials.

Taketoshi Matsumoto (Assoc Prof, Sci, PhD) received his MSc and PhD degrees electronic chemistry from Tokyo Institute of Technology, Yokohama, Japan in 2001. From 2000 to 2002 , he was a research fellow of the Japan Society for the Promotion of Science. Since 2001, he was a Postdoctoral Research Associate in University of Southern California, CA, USA. Since 2003, he was a Lecturer in University of Tsukuba, Tsukuba, Japan. Since 2004, he was a Research Associate in Institute for Molecular Science, Okazaki, Japan. Since 2007 and 2014, he has been an Assistant and Associate Professor, respectively, in the Institute of Scientific and Industrial Research, Osaka University, Osaka, Japan. He is interested in energy related nano-materials and devices such as TFTs, LSI, luminous materials, sealed permanent memories, solar cells and Li ion batteries.

Hikaru Kobayashi received the BE degree in chemistry from Osaka University, Osaka, Japan, in 1979 and the ME, and $\mathrm{PhD}$ degrees in chemistry from Kyoto University, Kyoto, Japan, in 1981, and 1984, respectively. From 1984 to 1986, he was a Postdoctoral Research Associate in Department of Physics and Astronomy, University of Pennsylvania, PA where he was engaged in spectroscopy on solid surfaces. From 19861987, he was a Researcher in Matsushita Electronics Corporation Kyoto Research Center, Kyoto, Japan where he was engaged in LSI wiring and gate oxide technology. From 1987 to 1990, he was a Research Associate in Department of Chemistry, Faculty of Engineering Science, Osaka University where he was engaged in research on silicon solar cells, surface and interface science of silicon, and low temperature silicon oxidation. From 1990 to 1998, he has been an Associate Professor in Department of Chemistry, Faculty of Engineering Science, Osaka University. He is currently a Professor in the Institute of Scientific and Industrial Research, Osaka University. He has been engaged in research on silicon solar cells, low temperature $\mathrm{Si}$ oxidation, and fabrication of Si nanopowder and its application to hydrogen generation material, anode material in $\mathrm{Li}$ ion batteries, and luminescent material. 